\title{
New Features of 3D Topological Order
}

Jeongwan Haah, "Local stabilizer codes in three dimensions without string logical operators", Phys. Rev. A 83, 042330 (2011)

Sergey Bravyi and Jeongwan Haah, "On the energy landscape of 3D spin Hamiltonians with topological order", arXiv:1105:4159

\section{Recommended and a Commentary by David DiVincenzo, RWTH, Aachen and FZ, Juelich.}

When it was invented, quantum error correction code theory didnt look like it had much to do with physics, certainly not with condensed matter physics. In the form that Shor introduced it in his nine-qubit code, with its quantum versions of parity checks, it seemed clearly the domain of communications theory. The stabilizer group theory that was subsequently discovered, which permitted a classification of large numbers of quantum error correction codes, looked like the domain of pure mathematics.

But these stabilizer groups have now been producing a steady stream of interesting physics ideas. The generators of a stabilizer group are multiqubit operators, a product of some Pauli spin operator for each qubit. The generators must all commute, and the common +1 eigenvectors of these operators are the code states of the quantum error correction code. But these code states also span the ground space of the exactly-solvable Hamiltonian formed as the sum of all these (commuting) generators.

Add geometric regularity, and you get some important quantum spin lattice models. The outstanding example is the $2 \mathrm{D}$ toric code, known to Wegner as an Ising lattice gauge theory decades ago, but explicated fully using the error correction machinery by Kitaev. The generators of the toric code are four-spin plaquette operators, products of Pauli-Z operators, defining already an interesting Ising-like model. But added to this is the same Ising model, defined on the dual lattice, but with $\mathrm{X}$ rather than $\mathrm{Z}$ as the Ising quantization axis.

The toric code has very good error correction properties: as long as errors do not percolate across the lattice, the code states are robust. In fact, these 
code states possess, and to some degree define, the notion of topological order: The system is gapped, with a ground state degeneracy that depends on the topology of the boundary conditions. The highly-entangled ground eigenstates are not distinguishable locally, and local operations cannot transform one into the other. Nor can the gap be closed by local disorder, unless it is very strong.

But the toric codes topological order does not prevent states in the ground space from being thermally fragile. The destruction of this quantum-memory property at finite temperature is explained by the phrase, logical operators are string-like, which requires some explanation. Logical operators are the (nonlocal) transformations that take the system from one ground state to another. At finite temperature, logical operators are generated by, first, the creation of excitations out of the vacuum. This costs a finite amount of energy (the gap) and results in two point-like quasiparticles (elementary examples of anyons). After creation, these particles can move without energy cost; there is no barrier to their going across the whole lattice. The path of this traversal defines the string that is the logical operator. Thus, the $2 \mathrm{D}$ toric code is inadequate as a passive memory for quantum information; it must be frequently error-corrected. Unfortunately, this is generic to two dimensions: with only short range interactions, it is impossible to build a macroscopic (i.e., growing with system size) barrier to the motion of pointlike excitations. The situation in four dimensions is very different: the 4D toric code has only membrane-like logical operators, and it has a passive retention time for quantum memory that scales favorably with system size.

And what about three dimensions? Some arguments had been abroad to indicate that stability was not possible there, and a sort of dimension counting has been shown to apply to a wide class of models, to the effect that the sum of the dimension of the logical operator plus that of its dual cannot exceed the space dimension. The toric code in even dimension is selfdual, and the rule works for these codes (string plus string $=2 \mathrm{D}$, membrane plus membrane $=4 \mathrm{D})$. The best candidate codes that had emerged in $3 \mathrm{D}$ were not self dual, and, sure enough, had both membrane and string logical operators. Enter Haah, with the thought that we should, with all the grouptheoretic machinery at our disposal from the code theorists, simply search, intelligently and painstakingly. Out from his search has popped a new 3D doubled-Ising-model code (several of them, in fact). All right, the resulting Hamiltonian has a particular form of 8-spin coupling, so it was not easy to come upon. But it is otherwise a perfectly respectable, translationallyinvariant spin Hamiltonian on a cubic lattice with short-range interactions. 
The phenomenology of the Haah model is unique. Pointlike excitations can be created, but are immobile; attempting to move them with local interactions leaves a cloud of wake excitations. With not much else that this, Bravyi and Haah show that the energy barrier to moving excitations across the lattice, and creating logical operators, is at least logarithmic in the system size. In Haahs model, the logical operators are in fact fractal, in retrospect a satisfying way of being reconciled with the sum rule above.

A scale-invariant landscape of energy barriers puts one in mind of spin glasses, and there are certainly relations between Haahs construction and the three-spin Ising model of Newman and Moore (Glassy dynamics and aging in an exactly solvable spin model, Phys. Rev. E 60, 5068 (1999)) . But it is entirely unanticipated that such structure could be doubled in the service of a full protection of quantum information. In the meantime, there is some neat quantum statistical mechanics still to be understood for the artifact that Haah has uncovered. 